\title{
THE REAL PART OF DECOMPOSITION OF A POLYNOMIAL AND ITS DETERMINACY
}

\author{
By Zhang Guobin and Sun Wei-Zhi
}

\section{Introduction}

Let $f(x, y), g(x, y):\left(\mathbb{R}^{2}, 0\right) \rightarrow(\mathbb{R}, 0)$ be two $C^{\infty}$ function-germs. Germs $f$ and $g$ are called to be $r$-jet equivalent if at $(0,0)$, their derivatives of degree not greater than $r$ are identical. Denote this fact by $j^{r}(f)=j^{r}(g)$. Germ $f$ is called to be $C^{0}-r$ determined if for each germ $g$ with $j^{r}(f)=j^{r}(g)$, there exists a germ of homeomorphism $h:\left(\mathbb{R}^{2}, 0\right) \rightarrow\left(\mathbb{R}^{2}, 0\right)$ such that $f \circ h=g . f$ is called to be $C^{0}$-finitely-determined if it is $C^{0}$-r-determined for some $r$. The degree of $C^{0}$-determinacy of $f$ is the least number such that $f$ is $C^{0}$-r-determined.

Germs $f$ and $g$ are called to be V-equivalent if germs $f^{-1}(0)$ and $g^{-1}(0)$ are homeomorphic.

Let $P_{0}(n, k ; \mathbb{R})$ denote the set of topological equivalence classes of germs of real polynomials in $n$ variables of degree $\leq k$, and $P_{0}(n, \mathbb{R})$ the set of those classes for all $k$. T. Fukuda [1] proved the Thom's conjecture: $P_{0}(n, k ; \mathbb{R})$ is a finite set. How about $P_{0}(n ; \mathbb{R})$ ? It is easy to see that $P_{0}(1 ; \mathbb{R})$ contains only three elements. For example, the germs $y=x^{2}$ and $y=x^{4}$ are $C^{0}$-equivalent (V.I. Arnol'd etc. [2], p. 12). In general, $y=x^{2 m}$ and $y=x^{2 n}$ belong to be the same class, and $y=x^{2 m+1}$ and $y=x^{2 n+1}$ belong to be the another class.

\section{Homogeneous case}

Let $P(x, y)$ be a germ of a real homogeneous polynomial of degree $k$. Then

$$
P(x, y)=a\left(x-b_{1} y\right) \cdots\left(x-b_{s} y\right)\left(x-c_{1} y\right) \cdots\left(x-c_{m} y\right)
$$

where $a, b_{i} \in \mathbb{R}, a \neq 0, c_{j} \in \mathbb{C}$. We have the following.

THEOREM 1. $P(x, y)$ is $C^{0}$-finıtely determıned if and only if $b_{i} \neq b_{j}$ for $i \neq j$. In this case, the degree of $C^{0}$-determinacy of $P$ is $k$.

THEOREM 2. Homogeneous polynomial-germs $P(x, y)$ and $Q(x, y)$ are $V$-equivalent if and only if they have the same number of real factors (do not account the repeated number, if $b_{i}=b_{j}$ for some $i, j$ ).

Remark. The degrees of $P$ and $Q$ may be unequal when they are V-equivalent. 
Corollary 3. $\quad P_{0}(n ; \mathbb{R})$ is infinıte for $n \geq 2$.

\section{Non-homogeneous case}

Let $F(x, y)$ be a germ of the following form

$$
F(x, y)=x^{n}+A_{1}(y) x^{n-1}+A_{2}(y) x^{n-2}+\cdots+A_{n}(y)
$$

where $A_{i}(y)$ is a real polynomial of $y$. By Newton-Puiseux Theorem,

$$
F(x, y)=\left(x-p_{1}(y)\right) \cdots\left(x-p_{s}(y)\right)\left(x-q_{1}(y)\right) \cdots\left(x-q_{m}(y)\right)
$$

where $p_{i}(y)$ is a real fraction power series in $y$ and $q_{j}(y)$ has some complex coefficients.

Remark. The coefficients of $p_{i}, q_{j}$ can be computed effectively out, so $p_{i}$ and $q_{j}$ are called the Puiseux roots.

THEOREM 4. If $p_{1}, \ldots, p_{s}$ are mutually distınct, then $F(x, y)$ is $C^{0}$-finitely-determined.

\section{Proofs}

Lemma 1 (Y.C. Lu [3], Theorem 2). Let $Z(x, y)=Z_{1} Z_{2} \cdots Z_{q}$, where $Z_{1}(x, y)$ is homogeneous of degree $a_{j}$ and the degree of $C^{0}$-determinacy of $Z_{j}$ is $k_{j}$. Moreover, $\left\{Z_{1}, Z_{2}, \ldots, Z_{q}\right\}$ is pairwıse relatively prime. Then $Z$ is $C^{0}$-m-determıned, where

$$
m=\max _{1 \leq_{i} \leq q}\left\{\sum_{\jmath=1}^{q} a_{\jmath}-a_{i}+k_{i}\right\} .
$$

Lemma 2 (T.C. Kuo [4], Corollary 1). Let $H(x, y)$ be homogeneous of degree $k$. If $H(x, y)=0$ is a non-singular projective variety, i.e. $\operatorname{grad} H(x, y)=0$ only when $x=$ $y=0$, then $H$ is $C^{0}$-k-determined.

Proof of Theorem 1. (1) Necessity. If $P$ has a real repeated factor, we have

$$
P=(x-a y)^{r} B(x, y), a \in \mathbb{R}, r \geq 2 .
$$

Then $x-a y$ is a comon factor of $\frac{\partial P}{\partial x}$ and $\frac{\partial P}{\partial y}$, so the line $x-a y=0$ is contained in $\left(\frac{\partial P}{\partial x}\right)^{-1}(0)$ and $\left(\frac{\partial P}{\partial y}\right)^{-1}(0)$, and $(0,0)$ is not an isolated critical point of $P$. By Bochnack and Lojasiewicz [5], $P$ is not $C^{0}$-finitely-determined.

(2) Sufficiency. If $P$ has no any real repeated factors, we have

$$
\begin{aligned}
P & =a \prod_{i=0}^{s}\left(x-b_{i} y\right) \prod_{\jmath=0}^{m}\left(x^{2}+c_{j} x y+d_{j} y^{2}\right)^{w_{\jmath}} \\
& =a Z_{1} Z_{2} \cdots Z_{s+m} . \quad \text { where } c_{\jmath}^{2}-4 d_{\jmath}<0, \quad j=1,2, \ldots, m .
\end{aligned}
$$

If $i \neq j, Z_{i}$ and $Z_{\jmath}$ are relatively prime. Let $g(x, y)=x-b y, b \in \mathbb{R}$. Obviously, $g$ is $C^{0}-1$ determined. Let $h(x, y)=\left(x^{2}+c x y+d y^{2}\right)^{t}, c^{2}-4 d<0, t \geq 1$, then $x^{2}+c x y+d y^{2}=1$ is an elliptic curve, so under new coordinate system, $h$ has the following form:

$$
h(x, y)=\left(x^{2}+y^{2}\right)^{t} .
$$


For $\frac{\partial h}{\partial x}=2 t x\left(x^{2}+y^{2}\right)^{t-1}, \frac{\partial h}{\partial y}=2 t y\left(x^{2}+y^{2}\right)^{t-1}$, then $\operatorname{grad} h=0$ only when $x=y=0$. By Lemma $2, h$ is $C^{0}$-k-determined.

Denote the degree of $Z_{\jmath}$ by $a_{\jmath}$, and the degree of $C^{0}$-determinacy of $Z_{\jmath}$ by $k_{j}$, then $a_{\jmath}=k_{j}$ for all $j$ from the above argument and $\sum_{\jmath=1}^{s+m} a_{\jmath}=k$. Hence by Lemma $1, P$ is $C^{0}$-k-determined.

Example. Let $P(x, y)=x^{5}+y^{5}$. Since $z^{5}+1=0$ has only one real root, so $P$ has only one real factor of its decomposition. By Theorem $1, P$ is $C^{0}-5$-determined. From D. Siersma [6] (p. 26), $P$ is $C^{\infty}$-6-determined. By Y.C. Lu [7] (p. 59), $P$ is not $C^{\infty}$-5-determined. This example and Theorem 1 show that for germs of homogeneous polynomials, the degree of $C^{0}$-determinacy is exactly the degree of polynomial if it is finite-determined, but it is not ture for the smooth case.

Proof of Theorem 2. In the express (A), $P(x, y)=0$ if and only if either $x-b_{i} y=0$ or $x^{2}+c_{1} x y+d_{j} y^{2}=0$ is satisfied. The curve $x-b_{i} y=0$ is a straight line $T_{i}$ passing through the origin, and the curve $x^{2}+c_{1} x y+d_{j} y^{2}=0$ contains only one point $(0,0)$, because $c_{\jmath}^{2}-4 d_{\jmath}<0$, then $P^{-1}(0)$ consists of lines $T_{\imath}$.
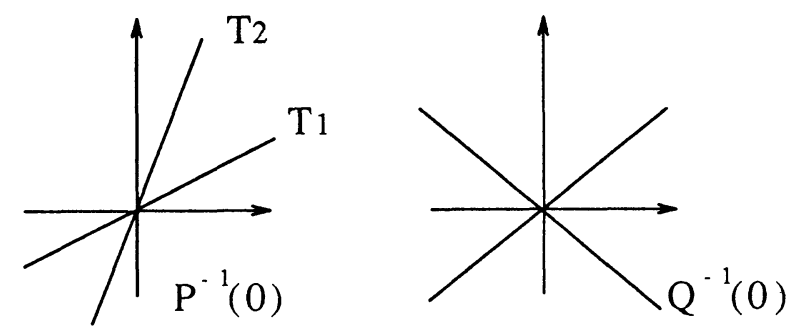

It is easy to see that $P^{-1}(0)$ and $Q^{-1}(0)$ have the same topological type if and only if they have the same number of lines $T_{\imath}$.

Proof of Corollary 3. For $n=2$, let $f_{k}(x, y)=(x-y)(x-2 y) \cdots(x-k y)$. By Theorem 2, $f_{2}$ is not $C^{0}$-equivalent to $f_{j}$ for $i \neq j$. For $n>2$, let $\bar{f}_{k}\left(x_{1}, \ldots, x_{n}\right)=\left(x_{1}\right.$ $\left.x_{2}\right) \cdots\left(x_{1}-k x_{2}\right)\left(x_{3}^{2}+\cdots+x_{n}^{2}\right)$, then $f_{k}\left(x_{1}, x_{2}\right)=\bar{f}_{k}\left(x_{1}, x_{2}, 0, \ldots, 0\right)$. Since $f_{i}\left(x_{1}, x_{2}\right)$ is not $C^{0}$-equivalent to $f_{j}\left(x_{1}, x_{2}\right)$ for $i \neq j$, hence $\bar{f}_{i}$ is not $C^{0}$-equivalent to $\bar{f}_{j}$.

Proof of Theorem 4. For any point $D$ near $(0,0)$,

$$
\begin{aligned}
\frac{\partial F}{\partial x} & =\sum_{i=1}^{s}\left[x-p_{1}(y)\right] \cdots\left(x-\hat{p}_{i}(y)\right) \cdots\left(x-p_{s}(y)\right)\left(x-q_{1}(y)\right) \cdots\left(x-q_{m}(y)\right) \\
& +\sum_{j=1}^{m}\left(x-p_{1}(y)\right) \cdots\left(x-p_{s}(y)\right)\left(x-q_{1}(y)\right) \cdots\left(x-\hat{q}_{j}(y)\right) \cdots\left(x-q_{m}(y)\right)
\end{aligned}
$$




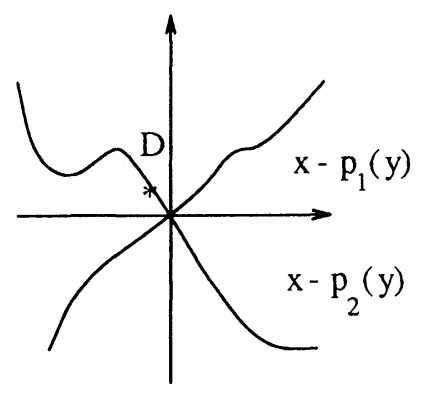

Therefore, $\frac{\partial F}{\partial x}=0$ at $D$ if and only if $D=(0,0)$, and so $(0,0)$ is the isolated critical point of $F$. By Kuo-Bochnack-Lojasiewicz Theorem, $F$ is $C^{0}$-finitely-determined.

The authors would like to thank Professor T.C.Kuo for his helpful talk about Theorem 4 .

\section{REFERENCE}

[1] T. Fukuda, Topologiques Des polynomes, I. H. E. S. Publ. Math. 46, (1976), 87-106.

[2] V.I. Arnol'd, S.M. Gusein-Zade and A.N. Varchenko, Singularities of Differentiable Maps, Vol. I, Birkhauser, Boston Inc., 1985.

[ 3 ] Yung-Chen Lu, Sufficiency of jets in $J^{r}(2,1)$ via Decomposition, Invent. Math. 10 (1970), 119127.

[4] T.C. Kuo, On $C^{0}$-sufficiency of jets of potential functions, Topology 8 (1969), 167-171.

[5] J. Bochnack and S. Lojasiewicz, A converse of the Kuiper-Kuo Theorem, LNM-Springer 192 (1971), 254-261.

[6] D. Siersma, Classification and deformation of singularities, Ph. D. thesis, Academis Service, Vinkveen (1974).

[ 7 ] Y.C. Lu, Singularity Theory and Introduction to Catastrophe, Springer-Verlag, New York, 1976.

[ 8 ] R.J. Walker, Algebraic Curves, Princeton Univ. press, 1950.

ZHANG GUOBIN

Department of Mathematics

Zhanjiang Teacher's College

Zhanjiang, Guangdong, 524048, P. R. China

SUN WEI-ZHI

Department of Mathematics

DONG Bei Normal University

Chang Chun, 130024, P. R. China 